\title{
MOLECULAR EPIDEMIOLOGY OF BRUCELLA MELITENSIS STRAINS CAUSING OUTBREAKS IN CROATIA AND BOSNIA AND HERZEGOVINA
}

\author{
Sanja DUVNJAK ${ }^{*}$, Ivana RAČIĆ, Silvio ŠPIČIĆ, Maja ZDELAR-TUK, Irena REIL \\ and Željko CVETNIĆ \\ Laboratory for Bacterial Zoonoses and Molecular Diagnosis of Bacterial Diseases, \\ Department for Bacteriology and Parasitology, Croatian Veterinary Institute, \\ Savska cesta 143, 10000 Zagreb, Croatia
}

(Received 23 November 2017; accepted 16 April 2018)

The most recent data on the incidence of brucellosis in Southeast Europe prove the persistence of this zoonosis in the area, regardless of constant efforts at controlling it as one of the most dangerous zoonoses. Forty-three Brucella melitensis strains were collected from cattle, sheep, goats and humans from Croatia as well as Bosnia and Herzegovina between 2009 and 2015. The strains were identified and genotyped in order to determine their epidemiological background. Standard biotyping methods and Bruce-ladder were used to identify the strains. Genotyping was done using multilocus variable number tandem repeats analysis (MLVA) on 16 and multilocus sequence typing analysis (MLST) on nine loci. Results were compared to each other and to internationally available data. Twenty-five novel genotypes and two sequence types were identified. All tested strains, apart from vaccine and reference strains, showed very close phylogenetic and geographic relationships. The genotyping results indicate the endemicity of brucellosis in this region. MLST showed no variation, confirming the stability of housekeeping genes. The results confirm already established routes of disease spread in this area, showing that a more detailed and vigorous control of this zoonosis is necessary.

Key words: Brucella melitensis, MLVA, MLST, Croatia, infectious disease

Brucellosis is a global zoonosis and one of the seven neglected, underreported and underdetected zoonotic diseases according to the World Health Organisation (WHO). It is endemic in Southeast Europe and the Mediterranean Region. Brucella melitensis has a very high prevalence in these countries and affects primarily sheep, goats and humans, but also cattle. The clinical signs usually include abortions in the last trimester of pregnancy, decreased productivity,

*Corresponding author; E-mail: sanja.duvnjak@gmail.com or marjanovic@veinst.hr; Phone: 00385 (1) 6123-635; Fax: 00385 (1) 6190-841 
weak offspring, and decreased milk production. This causes huge economic losses and is a major public health problem (Donev, 2010).

Sheep and goat farming plays a very significant role in the national economy of most developing countries, especially in the Balkan and Mediterranean Region. The low income level and nomadic nature of these regions makes the control and eradication of diseases extremely difficult (Ganter, 2015). In addition, these regions still carry a burden of recent and ongoing political changes, wars and new borders formed after Yugoslavia fell apart. Socioeconomic changes caused a reduction in monitoring and control funds, and their implementation became more challenging because of illegal cross-border trade of animals and their products. Combined with the intensified international migration of people, this caused a re-emergence of brucellosis in the last 15 years, changing it from a low-prevalence to an important endemic disease with high prevalence in sheep and goats as well as in humans (Donev, 2010).

The disease is still prevalent in Southeast Europe (especially in Macedonia, Turkey, Greece, Kosovo and Bosnia and Herzegovina) and in the Mediterranean Region (Italy, Portugal and Spain). Swine brucellosis is most prevalent in Croatia, but this country is one of the few Mediterranean countries having a favourable brucellosis status. However, financially and professionally well supported control and eradication programmes must be present to prevent this disease from becoming an even more serious problem than it had been reported to be in the past (Taleski et al., 2002; Pappas, 2010).

This research was conducted on B. melitensis strains isolated from sheep, goats, cattle and humans, originating from Croatia as well as Bosnia and Herzegovina. Strains were identified by biotyping and classical molecular approaches as well as genotyped using multilocus variable number tandem repeats analysis (MLVA-16) and multilocus sequence typing analysis (MLST). Results were compared to each other and to the Brucella MLVAbank international database (2016). The aim of the study was to define specificities and possible disease spread routes that could help elucidate the effectiveness of control programmes used at present and to guide them into possible new directions that may have appeared over the past decade.

\section{Materials and methods}

Bacterial strains tested. Forty-three B. melitensis strains isolated from different animal and human hosts and two countries over a period of six years, from 2009 to 2015, were used in this investigation. Most of the Croatian strains were isolated from collected tissue after routine testing and slaughter in three outbreaks ( 3 goats from Split-Dalmatia and one bovine from Karlovac County in 2009, 6 sheep from Lika-Senj and Split-Dalmatia counties and 3 goats from Split-Dalmatia county in 2010, 2 humans from Dubrovnik-Neretva County in 
2011, and one sheep from Lika-Senj County in 2013). Bosnia and Herzegovina (BIH) strains were gathered from materials sent to our laboratory by colleagues from the Veterinary Faculty of Sarajevo and the Veterinary Institute of Mostar from different parts of BIH ( 2 cattle in 2009; 5 cattle and 5 sheep from 2010; 12 cattle and 2 goats from 2012, and one human isolate from 2015). All isolates belonged to different animals or humans. In some cases more isolates from one animal were available but they were considered identical after biotyping and testing by classical molecular methods, and not all of them were genotyped for financial reasons. Two reference strains were used as control: one $B$. melitensis strain (16M) and a vaccine strain, Rev.1 (Table 1).

Table 1

Biotyping results according to host and origin

\begin{tabular}{lcc}
\hline Species and biovar & Host $^{1}$ & Origin \\
\hline Brucella melitensis biovar 1 & reference (2.22\%) & Slovenia \\
\hline Brucella melitensis Rev. 1 & sheep, reference (4.44\%) & Bosnia and Herzegovina, UNK ${ }^{2}$ \\
\hline & 20 bovine $(44.44 \%)$ & \\
Brucella melitensis biovar 3 & $\begin{array}{c}\text { 11 sheep }(24.44 \%) \\
\text { g goat }(17.78 \%)\end{array}$ & Croatia, Bosnia and Herzegovina \\
& 3 human $(6.67 \%)$ & \\
&
\end{tabular}

${ }^{1}$ strains are grouped according to the host from which they were isolated, presented as the number of animals as well as percentage of the total number of strains; ${ }^{2} \mathrm{UNK}$ - unknown origin

Biotyping. Strains were confirmed as Brucella using classical microbiological biotyping that included microscopic examination, culturing and biochemical tests (Corbel et al., 1983; Alton et al., 1988; OIE Manual, 2016).

Molecular diagnosis. Bruce-ladder, a multiplex PCR which differentiates most of the known Brucella species, was used as the reference method to determine Brucella species (Lopez-Goni et al., 2008).

Genotyping. MLVA-16 genotyping was done on a total of 16 gene loci (Le Flèche et al., 2006; Al Dahouk et al., 2007). Loci were divided into three panels: Panel 1 (Bruce06, 08, 11, 12, 42, 43, 45 and 55); Panel 2A (Bruce18 and 21) and Panel 2B (Bruce04, 07, 09, 16, 30 and Bruce19-previous Panel 2A member)(http://mlva.u-psud.fr/brucella/spip.php?article93; personal communication). Brucella melitensis $16 \mathrm{M}$ was used as the reference strain for comparison and verification of test quality. MLST was done on a total of 9 loci [gap, aroA, glk, dnaK, gyrB, trpE, cobQ, omp25, int-hyp (orf1)] according to Whatmore et al. (2007). 


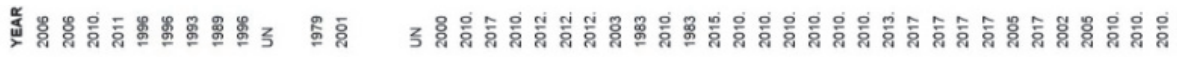

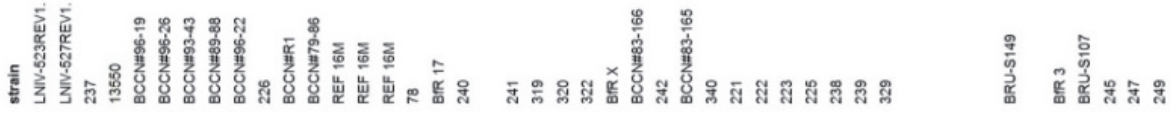

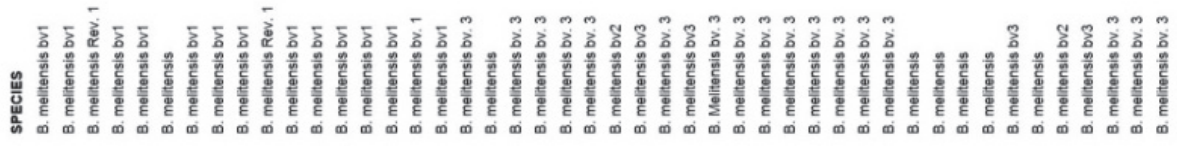

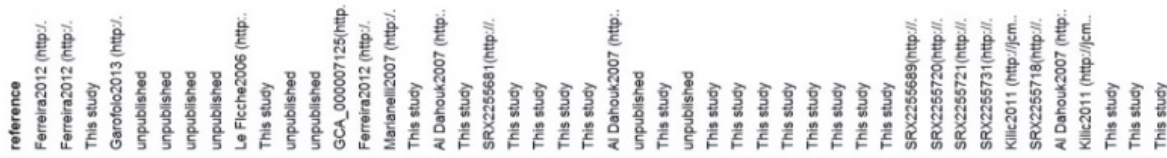

Whe

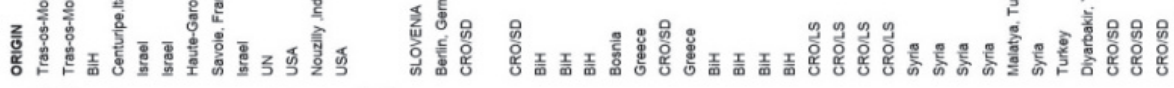

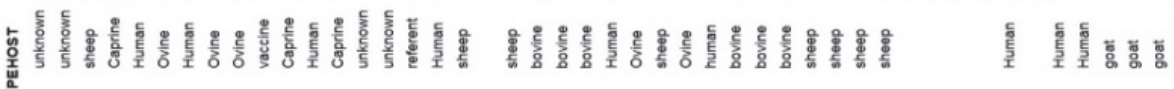

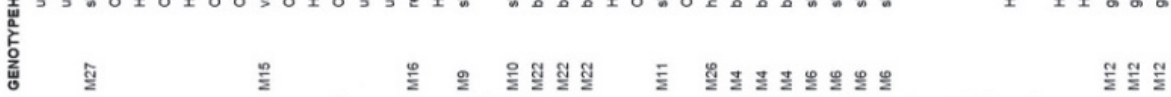

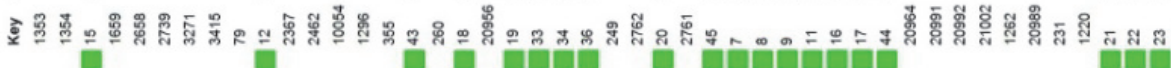

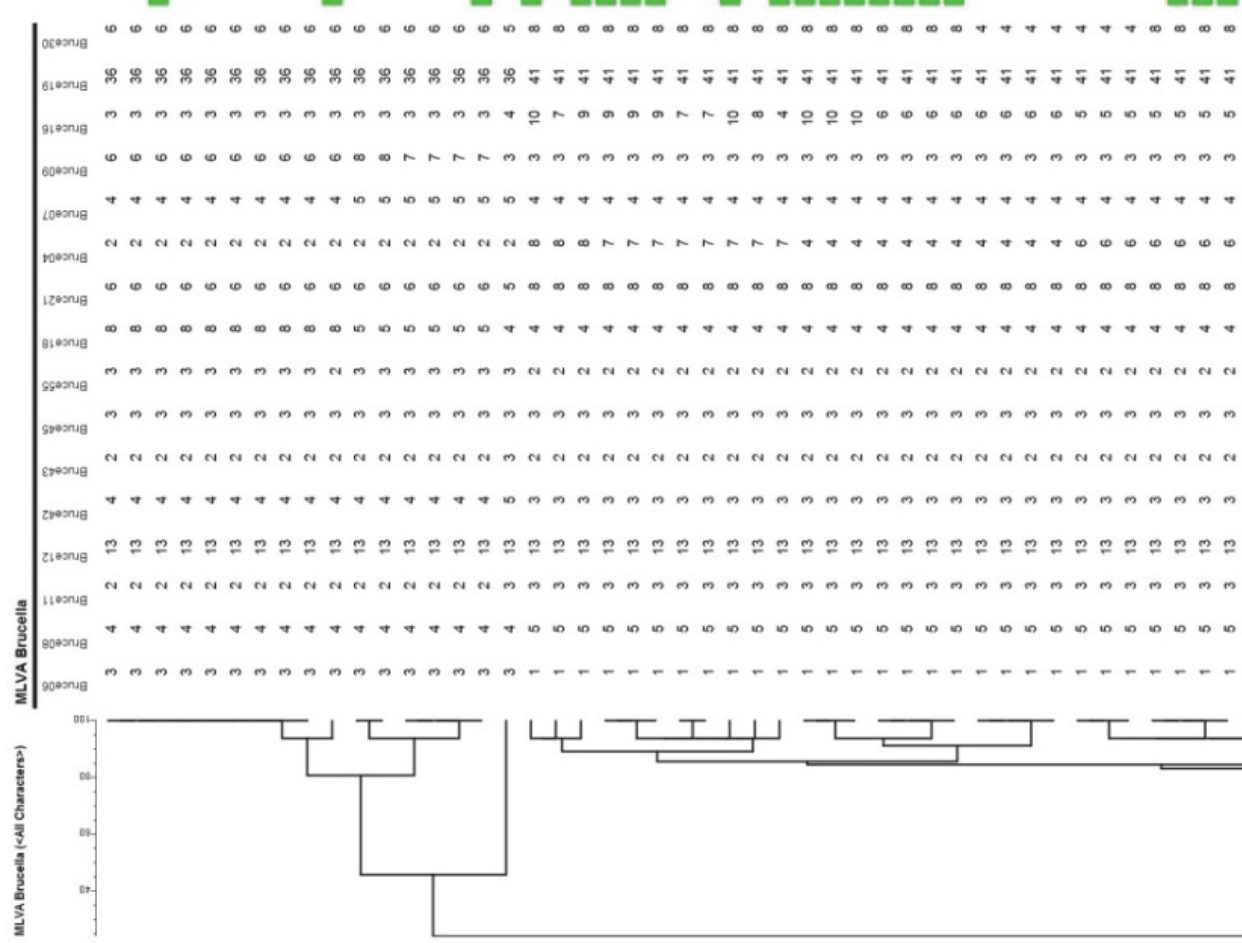

Acta Veterinaria Hungarica 66, 2018 


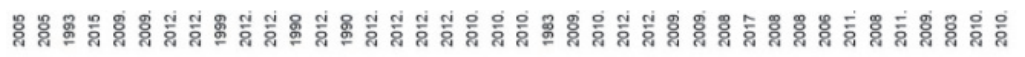

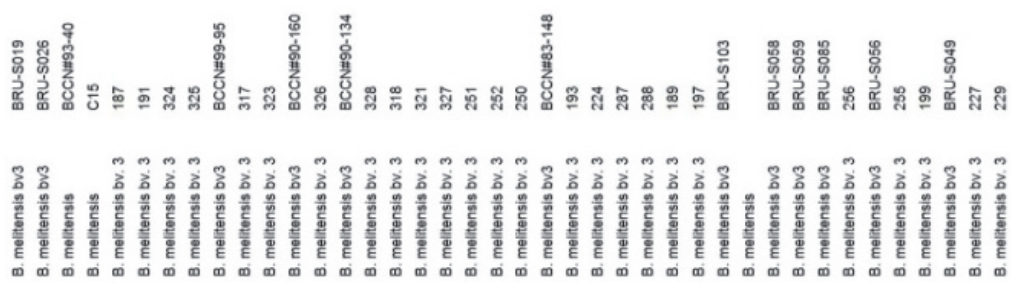

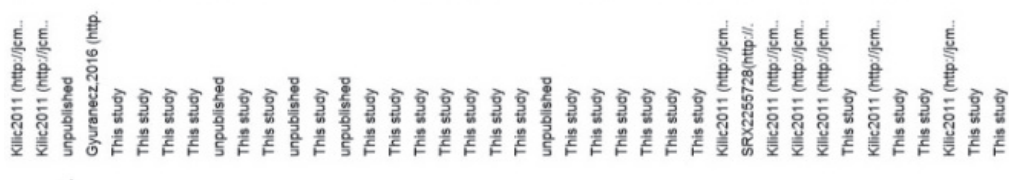

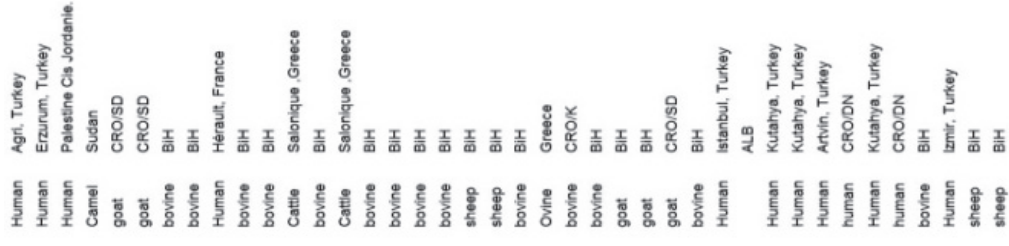

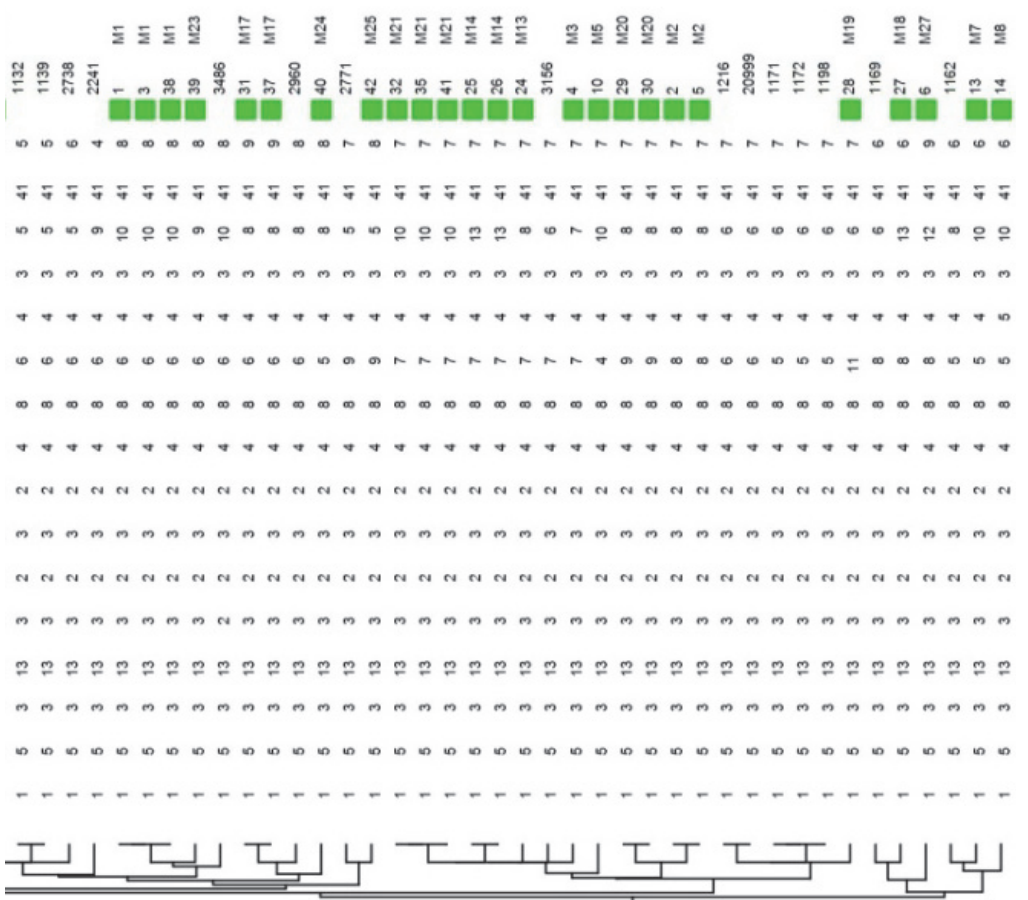


DNA was isolated using the commercially available QIAcube DNA Mini Kit and the QIAcube system (Qiagen, Hilden, Germany) according to the manufacturer's instructions. The supernatant $(2$ or $5 \mu \mathrm{L})$ was used in DNA-based tests.

The same PCR reaction mixture was used for all PCR reactions except for Bruce-ladder: $20-\mu \mathrm{L}$ reaction mixtures consisting of $10 \mu \mathrm{L}$ HotStarTaq Master Mix (Qiagen, Hilden, Germany), $6 \mu \mathrm{L}$ of water (RNase-free water, Qiagen, Hilden, Germany), $0.5 \mu \mathrm{M}$ of each primer pair specific for the target locus (Invitrogen, Paisley, UK or Macrogen, Amsterdam Zuid-Oost, Netherlands), and $2 \mu \mathrm{L}$ of template DNA. The cycling regime differed depending on the test but was done according to references (Le Flèche et al., 2006; Al Dahouk et al., 2007; Whatmore et al., 2007; Lopez-Goni et al., 2008). The 20- $\mu$ L PCR reaction mix for Bruce-ladder consisted of $10 \mu \mathrm{L}$ of QIAGEN Multiplex PCR Master Mix (Qiagen, Hilden, Germany), $2.5 \mu \mathrm{L}$ RNase-free water (Qiagen, Hilden, Germany), $0.4 \mu \mathrm{M}$ BMEI0998f and BMEI0997r primers (Invitrogen, UK or Macrogen, Netherlands), $0.1 \mu \mathrm{M}$ each of the remaining primers (Lopez-Goni et al., 2008), and $2 \mu \mathrm{L}$ DNA. All amplifications were performed on ProFlex PCR System (Applied Biosystems, USA). Amplification products were analysed using capillary electrophoresis on the QIAxcel system (Qiagen, Hilden, Germany) with size markers in the bp ranges of 100 to 2500 .

Band sizes from MLVA-16 results were translated into the number of individual repeats (Le Flèche et al., 2006). Results were presented in the form of 16-digit numerical codes based on version 3.6 Brucella allele assignment table (available at http://microbesgenotyping.i2bc.paris-saclay.fr).

Polymorphism was calculated using the Hunter-Gaston Diversity Index (HGDI) (Hunter and Gaston, 1988; Le Flèche et al., 2006). The obtained codes were analysed using the categorical coefficient and Unweighted Pair Group Method with Arithmetic Mean (UPGMA) using BioNumerics software (version 7.6; Applied Maths, Belgium). 'Fine-tuning' of markers' weight was suggested previously (Al Dahouk et al., 2007) but it must be done by comparing much more data in more detail from the same region in order to be of acceptable quality and specificity for this region.

Sequences from MLST were assembled, processed and compared using BioNumerics software (version 7.6; Applied Maths, Belgium). Alleles and sequence types were identified using profiles published by Whatmore et al. (2007) using BioNumerics. Alleles formed 9-digit numerical codes that were used in comparisons based on categorical coefficient and UPGMA.

Results from MLVA-16 and MLST were compared to results from different countries deposited in the Brucella MLVAbank (available at http://microbesgenotyping.i2bc.paris-saclay.fr) (Grissa et al., 2008). 


\section{Results}

We analysed 43 B. melitensis isolates from sheep, goats, cattle and humans to assess the epidemiological and geographic relationships of strains isolated over the past six years in Croatia and in Bosnia and Herzegovina (BIH), and their relationship to genotypes present in Europe.

Bacteriological typing (biotyping) identified 43 B. melitensis strains, mainly biovar 3 except for one Rev.1 sheep isolate originating from BIH in 2010. One biovar 1 (from Slovenia) and one Rev.1 vaccine strain were used as reference strains (Table 1).

Bruce-ladder results confirmed the results of biotyping. MLVA genotyping was more informative. MLVA-8 grouped all the strains in the East Mediterranean group. MLVA-11 revealed no new genotypes. MLVA-16 identified 27 genotypes, M1-M27 (Fig. 1). Twenty-five of those were novel genotypes deposited in the Brucella MLVAbank (http://microbesgenotyping.i2bc.paris-saclay.fr). These novel genotypes are not species specific but rather have geographic and outbreak specificity (Fig. 1). The HGDI evaluates the discriminatory power of the technique, which in turn suggests the power of a certain locus for differentiating specific strains. However, this is just an estimate because of strain to strain variations and due to the unknown influence of the host and geographic location. Only loci Bruce 04 and 16 showed high differentiating power, Bruce 30 showed moderate power, and the rest showed minimum or no power (Table 2).

The results were then compared to each other and to strains in the Brucella MLVAbank database. It is also important to mention that in the 3.6 version of the allele conversion table the coding convention for Bruce19 changed. This may cause possible misinterpretation of strain differences and differentiation of otherwise identical genotypes. This misinterpretation is not possible to verify (personal communication with a moderator of the MLVAbank at mlva.u-psud.fr).

The MLST data show that all biovar 3 isolates belong to ST8 and all biovar 1 isolates (including vaccine strains) belong to ST7 (Fig. 1).

\section{Discussion}

We identified and typed 43 B. melitensis strains in order to determine the background of the strains. Information like regional specificities of these strains, their similarities and differences could point to possible sources, weak points and directions of the spread of brucellosis.

Brucella melitensis causes brucellosis which is endemic in many countries, especially in Southeastern Europe and the Mediterranean Region. It has very high prevalence in these countries and affects primarily sheep, goats and humans, but lately also cattle. Clinical signs are specific and the disease causes 
considerable economic losses primarily because of lost trading opportunities but it is also a major public health problem because $B$. melitensis is the most contagious Brucella species for humans and causes a severely debilitating illness. It often goes misdiagnosed, neglected and unreported (Donev, 2010).

Table 2

Number of alleles and HGDI index at each locus and panel

\begin{tabular}{lccl}
\hline Locus & No. of alleles & Alleles & HGDI $^{\mathrm{a}}$ \\
\hline PANEL 1 & 13 & & \\
Bruce06 & 2 & 1,3 & 0.13 \\
Bruce08 & 2 & 4,5 & 0.09 \\
Bruce11 & 2 & 2,3 & 0.13 \\
Bruce12 & 1 & 13 & 0 \\
Bruce42 & 2 & 3,4 & 0.13 \\
Bruce43 & 1 & 2 & 0 \\
Bruce45 & 1 & 3 & 0 \\
Bruce55 & 2 & 2,3 & 0.09 \\
\hline PANEL 2A & 7 & $4,5,8$ & \\
Bruce18 & 3 & 35,40 & 0.13 \\
Bruce19 & 2 & 6,8 & 0.13 \\
Bruce21 & 2 & & 0.13 \\
\hline PANEL 2B & 27 & $4,8,5$ & \\
Bruce04 & 8 & $3,6,7$ & 0.84 \\
Bruce07 & 2 & $6,7,9,9,10,12,13$ & 0.09 \\
Bruce09 & 3 & $6,7,8,9$ & 0.85 \\
Bruce16 & 4 & & 0.65 \\
Bruce30 & 10 & $3,5,6,7,8,9,11$ & 0.22 \\
\hline
\end{tabular}

${ }^{\mathrm{a}}$ HGDI, Hunter-Gaston diversity index

In this study we identified strains of B. melitensis biovar 3, except for reference strains and one vaccine strain isolated from a sheep originating from $\mathrm{BIH}$. Biovars were determined by biotyping. To our knowledge, there is no other method, especially not a molecular one, suitable enough for biovar typing of $B$. melitensis. It would be logical to expect $B$. melitensis to be primarily isolated from sheep and goats, since these animals are its primary host. Twenty-four percent of the strains were isolated from sheep and $17.78 \%$ from goats. Somewhat less than 7 percent of the strains were isolated from humans. Forty-four percent were bovine isolates. Bovine brucellosis is not prevalent in Croatia (Cvetnic et al., 2014) and isolates from Croatia primarily originate from sheep and goats. This is not the case in $\mathrm{BIH}$, since $95 \%$ of our bovine samples were isolated from this region (Cvetnic et al., 2008; Spicic et al., 2010; Obradovic and Velic, 2010). 
This finding could be explained by the fact that vaccination had been practised for some time in BIH and the natural host for this bacterium has become unavailable. Brucellae could have been compelled to find another host by simple natural selection. However, this study does not contain sufficient information that could corroborate this assumption. Further, more detailed studies focused on the prevalence of brucellae applying systematic sample collection should be pursued in order to investigate the possible natural pressure that brucellae are under in these situations. One case of bovine brucellosis in Croatia was demonstrated in a cow from Karlovac County that borders with BIH. Such cases are almost always the result of illegal trade on both sides of the border.

Almost 98 (97.67\%) percent of the isolates were B. melitensis biovar 3 as it is the case in other Southeast European countries (Garofolo et al., 2013). We found one isolate from sheep that was a B. melitensis Rev.1 strain originating from BIH. The strain was isolated from an aborted lamb and the abortion had probably been induced by late administration of the vaccine. This strain is identical to some other vaccine strains present in the Brucella MLVAbank international database and differs from the reference vaccine strain in our own collection (of unknown origin) only at the Bruce55 locus. This is in agreement with findings from Garcia-Yoldi et al. (2007) that the Rev.1 vaccine strain is very stable over a long period of time.

Genotyping of strains was performed by two methods: MLVA on 16 loci and MLST on 9 loci (Le Flèche et al., 2006; Al Dahouk et al., 2007; Whatmore et al., 2007). MLVA-16 was able to identify 27 different genotypes out of which 25 were novel, according to the Brucella MLVAbank international database (Fig. 1). All the strains belong to the East Mediterranean (MLVA-8) group. No new MLVA-11 groups were identified. Only three loci show higher diversity (over 60\%): loci Bruce04, 16 and 30. All other loci show low or no diversity. This is just an estimate because not all loci carry the same weight (Al Dahouk et al., 2007). Strains of biovar 3 are distinguishable only on loci Bruce04, 16 and 30. However, even strains from the same outbreak, the same geographic region and the same year of isolation differ on some of these loci. Strains mutate very fast on these loci and thus these loci are not suitable for the long-term monitoring of $B$. melitensis strains. More samples, especially from countries with a high prevalence of infection like Bosnia and Herzegovina, need to be tested in order to be able to provide a 'fine-tuning' of markers' weight.

Strains are grouped in two major clusters (similarity over $80 \%$ ), one formed by biovar 1 strains, including Rev.1 strains and the other formed by biovar 3 strains, regardless of the origin of strains (Fig. 1). Unfortunately, this study is limited by the number and diversity of samples and, therefore, is not a good candidate for studying the suitability of MLVA-16 typing for biovar determination. All strains are very close phylogenetically, and diversity is below $20 \%$. The fact that our biovar 3 strains differ only at three loci that mutate very fast, 
even in the same outbreak, and not all of the strains were isolated from the same breeding facility, is a very probable proof of illegal trade on both sides of the border (Cvetnic et al., 2014). Strains are further grouped geographically and by outbreak. However, there are exceptions. There are two cases where strains with identical genotypes are three years apart. Strain number 44 was isolated from a sheep in Lika-Senj County in 2013 and has an identical genotype (M6) as three sheep isolates (numbers 11, 16 and 17) isolated from the same county in 2010. In the same way, a bovine isolate (number 38) obtained from BIH in 2012 has the same genotype (M1) as two goat isolates (numbers 1 and 3) from Split-Dalmatia County in 2009.

Two strains with identical genotypes appearing on two sides of the same border raise another important point. Namely, a goat isolate (number 2) from Croatia (Split-Dalmatia County) in 2009 and a bovine isolate (number 5) from the same year from BIH have an identical genotype. Also, cases appearing in Croatia are sporadic and low in prevalence, and emerging only in flocks and humans living in areas directly along the border with BIH. These findings support the assumption made by Cvetnic et al. (2014) that the illegal importation of animals from BIH is the main source of brucellosis in Croatia.

Considering human infection, we isolated and identified three human isolates, two from Croatia and one from BIH. There are no direct links to any of the outbreaks. Still, Croatian isolates from Dubrovnik-Neretva County, near the border with $\mathrm{BIH}$, were confirmed to originate from infections through the consumption of unpasteurised milk products.

The MLST results are quite uniform. All biovar 3 strains belong to ST8, while biovar 1 and vaccine strains belong to ST7. Therefore, this method in this form is not suitable for monitoring local infections in this region during a short period of time but could rather be used for monitoring large-scale outbreaks over a longer period of time (Whatmore et al., 2007).

Comparing our isolates with those deposited in the Brucella MLVAbank international database, most of the strains are in the closest relationship with strains originating from Turkey (the strains reported by Kilic et al., 2011) but also with strains from Greece and Syria (Fig. 1). One genotype (M12), apart from the $B$. melitensis $16 \mathrm{M}$ strain used by us as a reference strain, is identical to a Turkish human isolate (BRU-S107) obtained in 2005 (Fig. 1). These strains were isolated from goats originating from Split-Dalmatia County of Croatia, from two different farms. This supports the description provided by Pappas (2010), explaining how brucellosis spreads from Greece, Turkey, Macedonia, Albania, Kosovo and southern Serbia to Bosnia and Herzegovina, and Croatia.

In conclusion, this study has shown that biotyping and simple molecular methods are not suitable for monitoring the epidemiology and epizootiology of brucellosis. MLVA-16 proved effective in defining both the spread and the source of infection. The isolated strains are endemic for this region but are also 
phylogenetically very close to strains from Turkey, Greece and Syria. Using MLVA-16, this study again points to the high probability of illegal cross-border trading between Croatia and Bosnia and Herzegovina. The MLST method in this form is not suitable for monitoring the epidemiological situation of brucellosis in this region since the genes used do not mutate quickly enough. Also, this study raised the interesting question why $B$. melitensis so prevalently chooses a host different from its preferred host. All these findings point to the necessity of further studies involving a higher number of strains originating from all Balkan countries in order to be able to modify the control programmes accordingly. Studies applying systematic sample collection and incorporating much higher numbers of wildlife samples should be carried out in order to investigate the prevalence of brucellosis and to explain the host change. Also, additional efforts should be taken to stop the illegal or uncontrolled transfer of infected animals and their products across the region since illegal trading on both sides of the border is the most likely route of disease spread.

Understanding these findings is crucial not only for monitoring brucellosis in this region and throughout Southeast Europe but also for understanding how pathogens move across the continent and survive unfavourable conditions.

\section{Acknowledgements}

This work was supported by the Ministry of Science, Education and Sports of the Republic of Croatia (grant no. 048-0481153-1150).

\section{References}

Al Dahouk, S., LeFleche, P., Nöckler, K., Jacques, I., Grayon, M., Scholz, H. C., Tomaso, H., Vergnaud, G. and Neubauer, H. (2007): Evaluation of Brucella MLVA typing for human brucellosis. J. Microbiol. Methods 69, 137-145.

Alton, G. G., Jones, L. M., Angus, R. D. and Verger, J. M. (1988): Techniques for the brucellosis laboratory. Institut National de la Recherche Agronomique, Paris.

Corbel, M. J., Gill, K. P. W. and Thomas, E. L. (1983): Methods for the identification of Brucella. Central Veterinary Laboratory, New Haw, Weybridge, pp. 1-63.

Cvetnic, Z., Spicic, S., Kis, T., Zdelar-Tuk, M., Duvnjak, S., Racic, I., Benic, M., Habrun, B., Reil, I. and Sostar, Z. (2014): Brucellosis in the Republic of Croatia. Psych. Danubina 26, 546-551.

Cvetnic, Z., Velic, R., Spicic, S., Duvnjak, S., Zdelar-Tuk, M., Majnaric, D. and Aleraj, B. (2008): Distribution of brucellosis in the Republic of Croatia with an overview of the situation in Bosnia and Herzegovina. Croat. J. Infect. 28, 117-123.

Donev, D. M. (2010): Brucellosis control and eradication in the South Eastern European countries: Current status and perspective strategies. Mac. J. Med. Sci. 3, 220-227.

Donev, D. M. (2010): Brucellosis as priority public health challenge in South Eastern European countries. Cro. Med. J. 51, 283-284. http://doi.org/10.3325/cmj.2010.51.283.

Ganter, M. (2015): Zoonotic risks from small ruminants. Vet. Microbiol. 181, 53-65. 
García-Yoldi, D., Le Fleche, P., Marín, C. M., De Miguel, M. J., Muñoz, P. M., Vergnaud, G. and López-Goñi, I. (2007): Assessment of genetic stability of Brucella melitensis Rev 1 vaccine strain by multiple-locus variable-number tandem repeat analysis. Vaccine 25, 2858-2862.

Garofolo, G., Di Giannatale, E., De Massis, F., Zilli, K., Ancora, M., Camma, C., Calistri, P. and Foster, J. T. (2013): Investigating genetic diversity of Brucella abortus and Brucella melitensis in Italy with MLVA-16. Infect. Gen. Evol. 19, 59-70.

Grissa, I., Bouchon, P., Pourcel, C. and Vergnaud, G. (2008): On-line resources for bacterial micro-evolution studies using MLVA or CRISPR typing. Biochimie 90, 660-668.

Hunter, P. R. and Gaston, M. A. (1988): Numerical index of the discriminatory ability of typing systems: an application of Simpson's index of diversity. J. Clin. Microbiol. 26, 2465-2466.

Kiliç, S., Ivanov, I. N., Durmaz, R., Bayraktar, M. R., Ayaşlioğlu, E., Uyanik, M. H., Alişkan, H., Yaşar, E., Bayramoğlu, G., Arslantürk, A., Vergnaud, G. and Kantardjiev, T. V. (2011): Multiple-locus variable-number tandem-repeat analysis genotyping of human Brucella isolates from Turkey. J. Clin. Microbiol. 49, 3276-3283.

Le Flèche, P., Jacques, I., Grayon, M., Al Dahouk, S., Bouchon, P., Denoeud, F., Nöckler, K., Neubauer, H., Guilloteau, L. A. and Vergnaud, G. (2006): Evaluation and selection of tandem repeat loci for a Brucella MLVA typing assay. BMC Microbiol. 6, 9-22.

Lopez-Goni, I., Garcia-Yoldi, D., Marin, C. M., DeMiguel, M. J., Munoz, P. M., Blasco, J. M., Jacques, I., Grayon, M., Cloeckaert, A., Ferreira, A. C., Cardoso, R., DeSa, M. I. C., Walravens, K., Albert, D. and Garin-Bastuji, B. (2008): Evaluation of a multiplex PCR assay (Bruce-ladder) for molecular typing of all Brucella species, including the vaccine strains. J. Clin. Microbiol. 46, 3484-3487.

MLVA Bank for Microbes Genotyping on the internet page http://microbesgenotyping.i2bc.parissaclay.fr (accessed on $15^{\text {th }}$ August 2016).

Obradovic, Z. and Velic, R. (2010): Epidemiological characteristics of brucellosis in Federation of Bosnia and Herzegovina. Croat. Med. J. 51, 345-350.

OIE Manual of Diagnostic Tests and Vaccines for Terrestrial Animals (2016): Chapter 2.1.4. Brucellosis (Brucella abortus, B. melitensis and B. suis) (infection with Brucella abortus, $B$. melitensis and B. suis) http://www.oie.int/fileadmin/Home/eng/Health_standards/tahm/ 2.01.04_BRUCELLOSIS.pdf (cited 15.08.2016).

Pappas, G. (2010): The changing Brucella ecology: novel reservoir, new threats. Int. J. Antimicr. Agents 36, S8-S11.

Spicic, S., Zdelar-Tuk, M., Racic, I., Duvnjak, S. and Cvetnic, Z. (2010): Serological, bacteriological, and molecular diagnosis of brucellosis in domestic animals in Croatia. Croat. Med. J. 51, 320-326.

Taleski, V., Zerva, L., Kantardjiev, T., Cvetnic, Z., Erski-Biljic, M., Nikolovski, B., Bosnjakovski, J., Katalinic-Jankovic, V., Panteliadou, A., Stojkovski, S. and Kirandziski, T. (2002): An overview of the epidemiology and epizootology of brucellosis in selected countries of Central and Southeast Europe. Vet. Microbiol. 90, 147-155.

Whatmore, A. M., Perrett, L. L. and MacMillan, A. P. (2007): Characterisation of the genetic diversity of Brucella by multilocus sequencing. BMC Microbiol. 7, 34-48. 